\title{
Caracterización Epidemiológica de Pacientes con Infección Respiratoria Aguda (IRA) en un Hospital de Segundo Nivel en la Región Caribe colombiana Epidemiologic Profile of Patients with Acute Respiratory Infection in a Second Level Hospital at Colombian Caribean Region
}

Recibido: 11 Nov 2014 / Aceptado: 09 de Dic 2014

\author{
Lisbeth Martínez González ${ }^{1}$ \\ Universidad Simón Bolívar \\ José Narváez Barrios \\ Universidad Simón Bolívar. \\ Zenen Rua Osorio ${ }^{3}$ \\ Universidad Simón Bolívar \\ Wendy De Avila Barrera ${ }^{4}$ \\ Universidad Simón Bolívar \\ Inés Morales Salcedo 5 \\ Universidad Simón Bolívar \\ Ronald Maestre Serrano ${ }^{6}$ \\ Universidad Simón Bolívar
}

\section{RESUMEN}

Introducción: La Infección Respiratoria Aguda (IRA) es un evento de interés en salud pública para el departamento del Atlántico por su gran impacto en morbi-mortalidad. Objetivo: describir las características epidemiológicas de pacientes con IRA en el hospital Juan Domínguez Romero (JDR) del municipio de Soledad (Atlántico) durante el año 2013. Materiales y Métodos: Se realizó un estudio observacional, descriptivo, retrospectivo. La recolección de los datos se realizó a través de la revisión de las historias clínicas y las fichas de notificación epidemiológicas del Instituto Nacional de Salud en cada uno de los pacientes atendidos en urgencias y consulta externa del hospital JDR y que cumplieran con el criterio de inclusión durante el periodo de estudio. Resultados: Se analizaron 663 historias clínicas de pacientes con diagnóstico de IRA. El 57\% correspondieron a casos de infección respiratoria aguda grave, mientras que el $43 \%$ restante a enfermedad similar a la influenza. El 66,1\% de los pacientes con IRA se registraron durante el segundo semestre del año 2013; mientras que el 33,9\% restante durante el primer semestre. La mayoría de los pacientes eran del sexo hombre (52,2\%) y las etapas del desarrollo más frecuentes fueron: lactantes menores (36\%), adultez (13,7\%), adulto mayor $(11,8 \%)$, lactantes mayores $(11,6 \%)$ y preescolar (11\%). Los signos y síntomas más frecuentes en la población objeto de estudio fueron: tolerancia a vía oral $(70,9 \%)$, tos $(69,4 \%)$, fiebre $(69,2 \%)$ y dificultad respiratoria $(66,1 \%)$. Conclusión: Los resultados del presente trabajo contribuyen al conocimiento de las características epidemiológicas de las personas afectadas por esta enfermedad.

Palabras Clave: Enfermedad respiratoria, epidemiología, Colombia rehabilitación (Fuente: DeCS).

\section{ABSTRACT}

Introduction: Acute Respiratory Infection (ARIs) events as well as the morbidity and mortality associated to them are very important in Atlántico state. Objective: The aim of this study was making an epidemiologic characterization of the patients with ARIs at Juan Dominguez Romero (JDR) Hospitalin the town of Soledad (Atlántico), during 2013 Methodology: An observational, descriptive and retrospective study was conducted. The collection of data was performed reviewing clinical charts and notification cards of the National Institute of Health of every patient seen in the emergency department and the outpatient clinic that fulfill the inclusion criteria during the period of the study. Results: Analysis of 663 charts of patients with diagnosis of IRA was completed. Of these cases $57 \%$ were cases of severe IRA while the $43 \%$ were considered a disease similar to influenza. The $66.1 \%$ of the patients with IRA were registered during the second semester of 2013 and the $33.9 \%$ during the first semester. The majority of the patients were men (52.2\%). The developmental stages more often seen in patients with IRA were as follows: children under 1 year $(36 \%)$, adults $(13.7 \%)$, adults $>60$ years $(11.8 \%)$, children $12-23$ months $(11.6 \%)$, toddlers $2-5$ years $(11 \%)$. The signs and symptoms more frequently seen in the studied population were: nausea/vomiting $70.9 \%$, cough $69.4 \%$, fever $69.2 \%$ and respiratory distress $66.1 \%$. Conclusions: This study results represent a contribution to the knowledge about the epidemiologic characteristics of the population affected by this condition.

Keywords: Acute Respiratory Infection, epidemiology, Colombia.(Source: MeSH, NLM).

Para citar este artículo: Martínez González L, Narváez J, Rua Z. De Avila W, Morales I, Maestre R. Caracterización Epidemiológica de Pacientes con Infección Respiratoria Aguda (IRA) en un Hospital de Segundo Nivel en la Región Caribe colombiana. Cienc. innov. salud. $2014 ; 2$ (1):82 - 87

\footnotetext{
${ }^{1}$ Interno de Medicina - Universidad Simón Bolívar. E-mail: lismamago@ hotmail.com

${ }^{2}$ Interno de Medicina - Universidad Simón Bolívar. E-mail: jlelmono@ hotmail.com

${ }^{3}$ Interno de Medicina - Universidad Simón Bolívar. E-mail: zdrua@ hotmail.com

${ }^{4}$ Interno de Medicina - Universidad Simón Bolívar.

${ }^{5}$ ND., MPH., PhD Ciencias Sociales - Universidad Simón Bolívar. E-mail: Imorales4@ unisimonbolivar.edu.co

${ }^{6}$ Bió., PhD Medicina Tropical - Universidad Simón Bolívar. E-mail: rmaestre5@unisimonbolivar.edu.co
} 


\section{Introducción}

La Infección Respiratoria Aguda (IRA) es un evento de interés en salud pública a nivel mundial por su gran impacto en morbimortalidad, que afecta especialmente a países en vía de desarrollo, principalmente a los grupos poblacionales menores de cinco año, personas que cursan alguna enfermedad crónica de base y mayores de 60 años. Se estima que aproximadamente 4.000.000 de muertes por esta enfermedad se registran anualmente a nivel mundial(1). Este evento, contribuye a una alta carga económica debido a costos de atención en salud, pérdida de días laborales y escolares (2-4).

En Colombia la IRA es una de las principales causas de morbimortalidad en todos los grupos de edad, especialmente en los niños menores de 5 años y las personas mayores de 65 años $(1,4)$. Entre los años 2009 a 2011 se notificaronpara el país aproximadamente 14.440 .360 casos de IRA, de los cuales aproximadamente el $60 \%$ se registraron en población menor de 5 años (5). Los virus respiratorios causantes de IRA más frecuentemente identificados para Colombia son: Virus Sincitial Respiratorio, Influenza A/H1N1, influenza A/H3 estacional, Parainfluenza 3, Adenovirus, Influenza A e Influenza B y Parainfluenza 1 y 2 (5-10).

Las IRA es el primer evento de interés en salud pública que causa morbilidad en la población del departamento del Atlántico, los municipios más afectados por este evento son Barranquilla, Soledad, Malambo y Baranoa (11).Durante los años 2008 a 2010 el distrito de Barranquilla registró 80.202 casos de IRA con una tasa de incidencia entre 172 y 319 por cada 10.000 habitantes (Datos no publicados: Secretaria de salud distrital de Barranquilla). Hasta la semana epidemiológica 27 de los años 2011 a 2013 el sistema de vigilancia epidemiológica de la Secretaria de Salud del Atlántico registró 101.686 casos de IRA en población general de los 22 municipios restantes del departamento; de los cuales 40.808 (40\%) se registraron en población menor de cinco años (Datos no publicados: Secretaria de Salud del Atlántico). Para este departamento del país se ha identificado la circulación de los siguientes agentes causales: Influenza A, Virus Sincitial Respiratorio, Adenovirus y Parainfluenza 3(10, 12).

A pesar de la anterior situación, existen pocos estudios acerca de la epidemiología de este evento tanto en el departamento del Atlántico como en el municipio de Soledad; este último además de ser uno de los municipios con la mayor incidencia, concentra la segunda población más grande del departamento después de la capital Barranquilla. El objetivo del presente trabajo consistió en describir las características epidemiológicas de casos de IRA en el hospital Juan Domínguez Romero (JDR) del municipio de Soledad (Atlántico-Colombia) durante el año 2013.

\section{Materiales y Métodos}

El Departamento del Atlántico se encuentra ubicado al norte del territorio colombiano, entre los $11^{\circ} 37^{\prime} 16^{\prime \prime}$ y $10^{\circ} 16^{\prime}$ de latitud norte y los $74^{\circ} 43^{\prime}$ y $75^{\circ} 16^{\prime}$ de longitud occidental; cuenta con 23 municipios incluida su capital Barranquilla, tiene un área de $3.328 \mathrm{Km}^{2}$, una temperatura promedio de $28^{\circ} \mathrm{C}$ y humedad relativa de $70 \%$ aproximadamente (13).El estudio se realizó en el Hospital JDR del municipio de Soledad, que se encuentra localizado en las coordenadas geográficas $10^{\circ} 55^{\prime} \mathrm{N}$ y $74^{\circ} 46^{\prime} \mathrm{O}$. Limita por el norte con el distrito especial de Barranquilla, siendo el lindero el arroyo Don Juan; por el sur con el municipio de Malambo; por el oriente con el departamento del Magdalena, del cual está separado por el río Magdalena; y por el occidente con el municipio de Galapa.

Se realizó un estudio epidemiológico observacional, descriptivo, retrospectivo. La población participante se definió de la siguiente manera: Población general habitantes del área urbana y rural del municipio de Soledad (Atlántico) y municipios aledaños.

Se realizó un muestreo no probabilístico por conveniencia y la muestra correspondió a pacientes que acudieron a urgencias y consulta externa del hospital JDR, entre el 1 de enero y el 31 de diciembre de 2013, correspondiente a las semanas epidemiológicas 1 a 52 y que presentaron los siguientes signos y síntomas: manifestaciones clínicas leves con fiebre $>$ a $38^{\circ} \mathrm{C}$, tos $\mathrm{y}$ otros síntomas de tracto respiratorio superior de no más de siete días de evolución, o cualquier paciente con enfermedad respiratoria aguda que requirieron hospitalización y que presentaron fiebre $>$ a $38^{\circ} \mathrm{C}$, tos y dificultad respiratoria.

Se excluyeron todos los pacientes diagnosticados con rinitis alérgica, tosferina, sarampión, poliomielitis, fiebre tifoidea y otras enfermedades con síntomas similares a la IRA en su periodo inicial. 
Se estudiaron variables sociodemográficas tales como: sexo, edad, municipio y zona de residencia, estrato socioeconómico y régimen de seguridad social en salud; variables clínicasespecíficamente signos y síntomas y variables ambientales como precipitación pluvial y temperatura promedio ambiental para el municipio de Soledad (Atlántico) durante el año 2013. Las variables sociodemográficas y clínicas serecolectarona través de la revisión de las historias clínicas y la ficha de notificación epidemiológica individual $\mathrm{N}^{\circ} 345$ de Instituto Nacional de Salud, de cada uno de los pacientes atendidos en el hospital JDR que cumplieron con el criterio de inclusión durante el periodo de estudio. Se construyó una base de datos en el programa Excel que incluyó las variables estudiadas en cada uno de los pacientes. Para la descripción de éstas se calcularon las frecuencias absolutas y relativas. El estudio se realizó previa aprobación por parte del comité de ética de la Universidad Simón Bolívar y las directivas del hospital JDR

\section{Resultados}

Durante el periodo estudiado se analizaron 663 historias clínicas de pacientes que consultaron al hospital JDR con diagnóstico de IRA y que cumplieron con el criterio de inclusión establecido. De estos el 57\% (378/663) correspondieron a Infección Respiratoria Aguda Grave(IRAG) que requieren manejo intrahospitalario, mientras que el $43 \%$ (285/663) restante a Enfermedad Similar a la Influenza (ESI), cuyo manejo fue ambulatorio.

El 66,1\% (438/663) de los pacientes conIRA se registraron durante el segundo semestre del año 2013; mientras que el 33,9\% (225/663) restante durante el primer semestre (Tabla1).

El 98,9\% (656/663) de los pacientes con IRA que consultaron en el hospital JDR durante el periodo estudiado residían en el municipio de Soledad; sin embargo, el 1,1\% (7/663) restante residían en los municipios de Barranquilla, Malambo y Palmar de Varela en el departamento del Atlántico. Del total de pacientes estudiados, el 99,5\% residían en área urbanas y el 0,5\% en áreas periurbanas y rurales de los municipios anteriormente mencionados. El 79\% (524/663) de los casos de IRA correspondieron a personas que residían en barrios de estrato I y el $21 \%$ (137/663) restante en barrios de los estratos II y III (Tabla 1).
Tabla 1 Principales características sociodemográficas de pacientes con IRA que consultaron al Hospital JDR del municipio de Soledad en el departamento del Atlántico (Colombia), durante el año 2013.

\begin{tabular}{|c|c|c|c|c|c|c|}
\hline \multirow{2}{*}{ Variables } & \multirow{2}{*}{$\begin{array}{l}\text { ESI } \\
\mathbf{N}\end{array}$} & \multicolumn{2}{|c|}{ IRAG } & \multicolumn{3}{|c|}{ Total } \\
\hline & & $\%$ & $\mathbf{n}$ & $\%$ & $\mathbf{N}$ & $\%$ \\
\hline Pacientes & 285 & 43,0 & 378 & 57,0 & 663 & 100 \\
\hline \multicolumn{7}{|l|}{ Sexo } \\
\hline Hombre & 150 & 22,6 & 196 & 29,6 & 346 & 52,2 \\
\hline Mujer & 135 & 20,4 & 182 & 27,5 & 317 & 47,8 \\
\hline Etapa del Desarrollo & $\mathbf{N}$ & $\%$ & $\mathbf{n}$ & $\%$ & $\mathbf{N}$ & $\%$ \\
\hline Neonato (1 a 29 días) & 0 & 0 & 1 & 0,2 & 1 & 0,2 \\
\hline $\begin{array}{l}\text { Lactante menor ( } 30 \\
\text { días a } 11 \text { meses) }\end{array}$ & 103 & 15,5 & 139 & 21,0 & 242 & 36,5 \\
\hline $\begin{array}{l}\text { Lactante mayor ( } 12 \text { a } \\
23 \text { meses) }\end{array}$ & 25 & 3,8 & 52 & 7,8 & 77 & 11,6 \\
\hline $\begin{array}{l}\text { Pre-escolar ( } 2 \text { a } 5 \\
\text { años) }\end{array}$ & 35 & 5,3 & 38 & 5,7 & 73 & 11,0 \\
\hline Escolar (6 a 12 años) & 19 & 2,9 & 24 & 3,6 & 43 & 6,5 \\
\hline $\begin{array}{l}\text { Adolescencia (12 a } 17 \\
\text { años) }\end{array}$ & 14 & 2,1 & 14 & 2,1 & 28 & 4,2 \\
\hline $\begin{array}{l}\text { Juventud (18 a } 25 \\
\text { años) }\end{array}$ & 13 & 2,0 & 17 & 2,6 & 30 & 4,5 \\
\hline Adultez (26 a 60 años) & 43 & 6,5 & 48 & 7,2 & 91 & 13,7 \\
\hline $\begin{array}{l}\text { Adulto mayor(> } 60 \\
\text { años) }\end{array}$ & 33 & 5,0 & 45 & 6,8 & 78 & 11,8 \\
\hline $\begin{array}{l}\text { Municipio de } \\
\text { Residencia }\end{array}$ & $\mathbf{N}$ & $\%$ & $\mathbf{n}$ & $\%$ & $\mathbf{N}$ & $\%$ \\
\hline Soledad & 281 & 42,4 & 375 & 56,6 & 656 & 98,9 \\
\hline Otro municipio & 4 & 0,6 & 3 & 0,5 & 7 & 1,1 \\
\hline Zona de Residencia & $\mathbf{N}$ & $\%$ & $\mathbf{n}$ & $\%$ & $\mathbf{N}$ & $\%$ \\
\hline Urbana & 285 & 43,0 & 375 & 56,6 & 660 & 99,5 \\
\hline Periurbana & 0 & 0 & 1 & 0,2 & 1 & 0,2 \\
\hline Rural & 0 & 0 & 2 & 0,3 & 2 & 0,3 \\
\hline $\begin{array}{l}\text { Estrato Socio- } \\
\text { económico }\end{array}$ & $\mathbf{N}$ & $\%$ & $\mathbf{n}$ & $\%$ & $\mathbf{N}$ & $\%$ \\
\hline $\mathrm{I}$ & 212 & 32,0 & 312 & 47,1 & 524 & 79,0 \\
\hline II & 68 & 10,3 & 64 & 9,7 & 132 & 19,9 \\
\hline III & 3 & 0,5 & 2 & 0,3 & 5 & 0,8 \\
\hline NS/NR & 2 & 0,3 & 0 & 0 & 2 & 0,3 \\
\hline $\begin{array}{l}\text { Régimen de } \\
\text { Seguridad Social en } \\
\text { Salud }\end{array}$ & $\mathbf{N}$ & $\%$ & $\mathbf{n}$ & $\%$ & $\mathbf{N}$ & $\%$ \\
\hline Contributivo & 13 & 2,0 & 7 & 1,1 & 20 & 3,0 \\
\hline Subsidiado & 250 & 37,7 & 351 & 52,9 & 601 & 90,6 \\
\hline Vincualdo & 20 & 3,0 & 20 & 3,0 & 40 & 6,0 \\
\hline NS/NR & 2 & 0,3 & 0 & 0 & 2 & 0,3 \\
\hline
\end{tabular}
pacientes diagnosticados con ESI/IRAG,
ESI/IRAG; NS/NR: No Sabe/No Responde.

El $52,2 \%(346 / 663)$ de los casos de IRA se registraron en hombres y el 48,8\% (317/663) restante en mujeres. Las etapas del desarrollo más frecuentes en los pacientes con IRA fueron las siguientes: lactante menor $(36 \%)$, adultez $(13,7 \%)$, adulto mayor $(11,8 \%)$, lactante mayor $(11,6 \%)$ y preescolar $(11 \%)$ (Tabla 1).

El 90,6\% (601/663) de los pacientes de IRA pertenecían al régimen de salud subsidiado, mientras que el $9,4 \%$ restante a los regímenes contributivo y vinculado del sistema de seguridad social en salud. 
Entre los signos y síntomas más frecuentes en la población objeto de estudio se encontraron: tolerancia vía oral $(70,9 \%)$, tos $(69,4 \%)$, fiebre $(69,2 \%) \mathrm{y}$ dificultad respiratoria $(66,1 \%)$ (Figura 1).

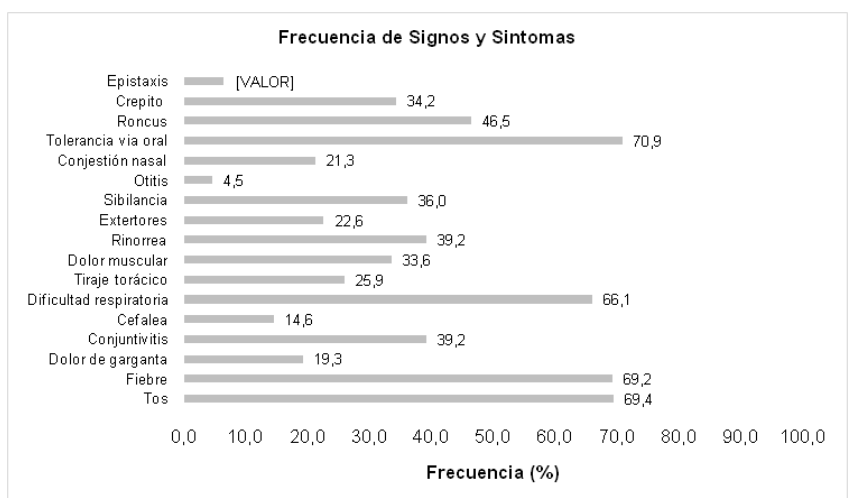

Figura 1 Frecuencia de signos y síntomas de pacientes con IRA que consultaron al Hospital JDR del municipio de Soledad en el departamento del Atlántico (Colombia), durante el año 2013.

La mayoría de los pacientes con IRA que consultaron al hospital JDR, durante el año 2013 gozaban de buena salud; sin embargo, se encontró que el 11,3\% (75/663) de estos pacientes manifestaron tener antecedentes de asma, el 2,1\% (14/663) enfermedad cardiaca, el 2\% (13/663) enfermedad pulmonar obstructiva crónica (EPOC), diabetes y obesidad respectivamente y el $0,2 \%(1 / 663)$ VIH y otras inmunodeficiencias. En cuanto a factores de riesgo el 6\% (40/663) manifestó tener hábitos de fumar y el $0,2 \%$ (1/663) tomar medicamentos inmunosupresores.

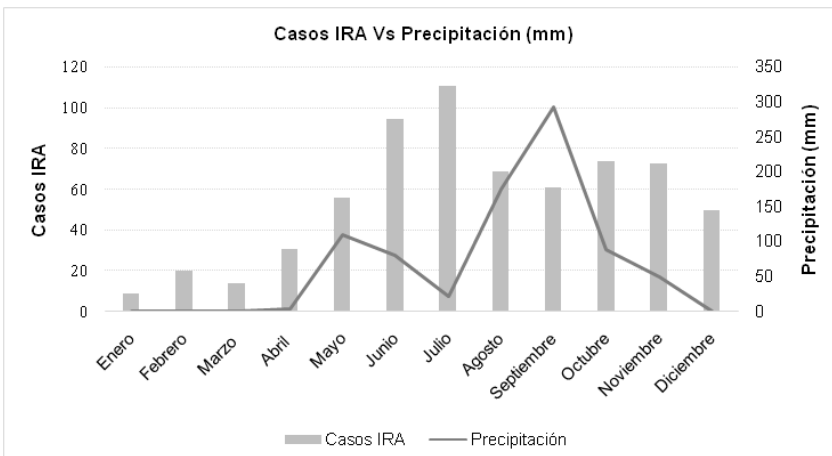

Figura 2 Comportamiento de casos de IRA del hospital JDR y precipitación pluvial en el municipio de Soledad (Atlántico), durante el año 2013. Fuente: Instituto de Hidrología, Meteorología y Estudios Ambientales de Colombia (IDEAM)

Durante los tres primeros meses del año 2013, cuando no se registraron lluviasse observóbaja frecuencia de casos de IRA. Durante los meses de abril y mayo aumentaron considerablemente, y durante estos meses se registraron las primeras lluvias del año. Sin embargo, la frecuencia de casos de IRA fue mayor en los meses de agosto a noviembre, donde se registró las mayores precipitaciones para el departamento del Atlántico (49,4 - 293,2 mm) (Figura 2). En la figura 3 se observa que durante los meses de junio y julio del año 2013 se registró las mayores temperaturas promedios para el departamento del Atlántico (28,5 y $28,8^{\circ} \mathrm{C}$ respectivamente); así mismo durante estos dos meses se registró el mayor número de casos de IRA.

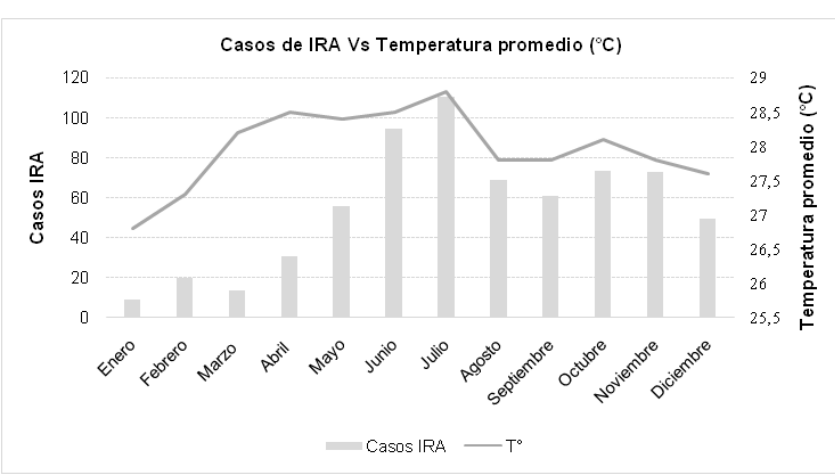

Figura 3Comportamiento de casos de IRA del hospital JDR y temperatura promedio en el municipio de Soledad (Atlántico), durante el año 2013. Fuente: Instituto de Hidrología, Meteorología y Estudios Ambientales de Colombia (IDEAM)

\section{Discusión}

Los resultados obtenidos en el presente trabajo de investigación, indicaron que durante el año 2013 se registró mayor frecuencia de casos de IRAG, con respecto a casos de ESI. Lo anterior, coincide con otros estudios reportados en el país (5)y puede estar relacionado a que las formas graves de la enfermedad son las que consultan por la atención medica que requieren; adicionalmente más del $70 \%$ de los casos de IRA en nuestro estudio se presentaron en los grupos etareos menores de 5 años y mayores de 60 años, los cuales son los grupos poblacionales más susceptibles debido a la relativa inmunidad inmadura en los menores de cinco años y a la inmunocompetencia en mayores de 60 años. Estos grupos poblacionales también han sido identificados como los más afectados por IRA en otros estudios realizados en Colombia y en otros países del mundo(5, 14-16).En Colombia para la población menor de cinco años se ha identificado el área de residencia, lactancia materna, anemia nutricional, uso de servicios de salud a través del carné de vacunación y la riqueza del hogar como determinantes sociales que inciden en la prevalencia de IRA (17). 
El comportamiento de los casos de IRAG con respecto a los casos de ESI también puede estar relacionado con el o los agente(s) etiológico circulante(s), ya que se ha demostrado que algunos de los virus respiratorios son estacionales y algunos de estos generan mayores complicaciones de la enfermedad (7, 18-19); Sin embargo, este aspecto fue una limitante del estudio ya que nuestro alcance no incluyó la identificación de agentes causales, lo cual hubiese podido aclarar un poco más la información con respecto a las complicaciones de la enfermedad en los pacientes con IRA estudiados. En Colombia los virus más frecuentemente identificados como agente causal de IRA incluye a virus sincitial respiratorio, adenovirus, influenza A y B, parainfluenza 1, 23 (69). En el departamento del Atlántico similares resultados se han encontrado con respecto al resto del país, ya que los virus respiratorios más frecuentes para este departamento son:influenza A, virus sincitial respiratorio, adenovirus y parainfluenza $3(10,12)$ y al igual que en otras investigaciones nuestros resultados coinciden con los reportados en diferentes regiones del país al identificar la tos, fiebre y dificultad respiratoria como los síntomas más frecuentes en estos pacientes (19). En los resultados obtenidos se encontró muy baja frecuencia de enfermedades crónicas de base en los pacientes con IRA; sin embargo, estudios a nivel mundial han indicado que estas enfermedades de base se asocian como predictores de severidad y mortalidad en casos de IRAG (20).

Los resultados obtenidos en el presente estudio indican que la IRA se presenta en mayor frecuencia en hombres que en mujeres coincidiendo con otros estudios(5). La mayoría de la población afectada con IRA que consultó al hospital JDR durante el año 2013 vivían en el área urbana del municipio de Soledad, teniendo en cuenta que este hospital es una de las principales instituciones de saludde la red pública del municipio, el cual atiende en su gran mayoría población pobre no asegurada, lo cual es coherente con los resultados obtenidos ya que la mayoría de la población afectada por IRA pertenecían al régimen de salud subsidiado y a los estratos I y II.

Al analizar los casos de IRA con respecto a las variables ambientales de precipitación se observó que existe un comportamiento directamente proporcional. El departamento del Atlántico presenta una distribución de precipitaciones de tipo monomodal cuyo periodo va de mayo a octubre siendo el mes de septiembre el de mayor precipitación. Durante este periodo de tiempo se registró el $70 \%$ de los casos de IRAG en el hospital JDR lo que coincide con otros estudios realizados en países de la región que se caracterizan por tener un régimen de lluvia similar (21). En otros países tropicales a nivel mundial se ha relacionado que la mayoría de los casos de IRA se registran en los periodos de mayor precipitación y altas temperaturas $(18,22)$.

Con los resultados del presente trabajo de investigación se amplía la información acerca de las características epidemiológicas y clínicas de la IRA en el municipio de Soledad en el departamento del Atlántico, como componente fundamental para el diseño de estrategias de promoción y prevención.

\section{Agradecimientos}

Expresamos agradecimientos al hospital JDR del municipio de Soledad por avalar y permitir la ejecución del presente estudio y a la Dra. Soraya Salcedo Mendoza por el apoyo en las asesorías brindadas.

\section{Conflicto de interés}

Los autores declaramos que no existe conflicto de intereses sobre los resultados aquí presentados..

\section{Referencias Bibliográficas}

1. Instituto Nacional de Salud (INS). Protocolo de vigilancia en salud pública de la Infección Respiratoria Aguda (IRA). Bogotá-Colombia, 2014:1-48 pp.

2. Louie JK, Hacker JK, Gonzales R, Mark J, Maselli JH, Yagi S, et al.Characterization of Viral Agents Causing Acute Respiratory Infection in a San Francisco University Medical Center Clinic during the Influenza Season. Clinical Infectious Diseases. 2005; 41(6):822-28.

3. Porras RA, Alvis GN, Rico MA, Alvis EL, Castañeda OC, Velandia GM, et al. Costo efectividad de la vacuna contra influenza en menores de dos años y mayores de 65 años en Colombia. Revista deSalud Pública.2009; 11(5):689-99.

4. Pulgarín TA, Osorio GS, Restrepo SY, Segura CM. Conocimientos y prácticas del cuidador como factor asociado a enfermedad respiratoria aguda en

Cienc. innov. salud. Diciembre 2014; 2 (2):82 - 87. Universidad Simón Bolívar (Col).ISSN: 2344-8636 http://portal.unisimonbolivar.edu.co:82/rdigital/innovacionsalud 
niños de 2 meses a 5 años. Investigación y Educación en Enfermería.2011; 29(1):19-27.

5. Castillo RL. Comportamiento de la Infección Respiratoria Aguda en Colombia, 2010. Informe Quincenal Epidemiológico Nacional.2011; 16(23):302-16.

6. Herrera D, De la Hoz F, Mariño C, López JD, Vélez C, Arboleda LM. Vigilancia de influenza y otros virus respiratorios en Colombia, enero del 2000 a 31 de julio de 2001. Informe Quincenal Epidemiológico Nacional. 2001;6(17):253-58.

7. Herrera D, De la Hoz F, Mariño C, Ramírez E, López J, Vélez C. Adenovirus en menores de cinco años: Patrones de circulación, características clínicas y epidemiológicas en Colombia, 19972003. Revista de Salud Pública.2007; 9(3):420-29.

8. Herrera D, De la Hoz F, Velandia M. Severe respiratory disease and its relationship with respiratory viruses in Colombia. International Journal of Infectious Disease.2008; 12:139-42.

9. Rojas LJ, Jaramillo CA, Mojica MJ, Escalante MP, Delgado P. Molecular typing of adenovirus circulating in a Colombian paediatric population with acute respiratory infection. Epidemiology and infection. 2012; 140:818-22.

10.Piñeros JG, Baquero H, Bastidas J, García J, Ovalle J, Patiño C, et al. Respiratory Syncytial virus infection as a cause of hospitalization in population under 1 year in Colombia.Journal of Pedriatrics. 2013;89(6):544-48.

11.Navarro LE, Barceló MR, Tuesca MR. Análisis de la situación de salud del departamento del Atlántico, 2004-2005. Ediciones Uninorte. Barranquilla-Colombia.2008. 354 pp.

12. Guerra M, Vergara C, Sibaja E, Anaya D, Maestre R. Vigilancia centinela para influenza y otros virus respiratorios en el departamento del Atlántico durante el periodo comprendido entre enero y julio de 2007. Biomédica.2007; 27(2):269.

13.Instituto Geográfico Agustín Codazzi (IGAC). Características geográficas del Departamento del Atlántico. Bogotá-Colombia, 1994.

14.Ferreira GE, Báez SR, Trejo VB, Ferreyra RL, Delgado SG, Chilián HOL, et al. Infecciones respiratorias agudas en niños y signos de alarma identificados por padres y cuidadores en México. Revista de Salud Pública deMéxico.2013; 55(2):307-13.

15.Rimmelzuaan G, Fouchier R, Osterhaus A. Age distribution of cases caused by different influenza viruses. Lancet Infectious diseases.2013; 13:64647.

16.Terajima M, Dawn M, Ennis F. Age and different influenza viruses. Lancet Infectious diseases.2014; 14:101.

17.Arrieta FR, Caro GEE. Determinantes sociales y disparidades de la morbilidad por enfermedades respiratorias agudas en niñas y niños de uno a cuatro años en Colombia. Revista gerencia y políticas en salud. 2010; 9(18):137-56.

18. Shek LP, Lee BW. Epidemiology and seasonality of respiratoy tract virus infections in the tropics. Paediatric Respiratory Reviews. 2003; 4:105-11.

19. Cotes K, Moreno MJ, Porras RA, Rico MA, De la Hoz RF. Características clínicas de pacientes hospitalizados con enfermedad respiratoria severa durante temporadas de influenza en las ciudades de Bogotá y Manizales, Colombia 2000-2006. Revista de Salud Pública.2012; 14(1):129-42.

20.Rodríguez DA, Rodríguez MC, Cárdenas AC, Quilaguy IE, Mayorga LY, Falla LM, et al. Predictors of severity and mortality in children hospitalized with respiratory sincytial virus infection in a tropical region. Pediatric Pulmonology.2014; 49(3):269-76.

21.Verani J, McCracken J, Arvelo W, Estevez A, Lopez M, Reyes L, et al. Surveillance for hospitalized acute respiratory infection in Guatemala. Plos One.2013; 8(12):e83600. doi: 10.1371/journal.pone.0083600. eCollection 2013.

22.Noguera S, Karchmer KS, Esilrabadan MC, Sánchez PA. Informe del primer caso de defunción secundaria a una nueva cepa humana del virus de la influenza A H1N1 en el mundo y comportamiento de la influenza humana en mujeres embarazadas. Ginecología y Obstetricia de Mexico.2013; 81:47-5 